\title{
Down-regulation of miR-210-3p encourages chemotherapy resistance of renal cell carcinoma via modulating ABCC1
}

\author{
Songchao Li $i^{1,2}$, Jinjian Yang ${ }^{1,2}$, Jun Wang ${ }^{1,2}$, Wansheng Gao ${ }^{1,2}$, Yafei Ding ${ }^{1,2}$, Yinghui Ding ${ }^{1,2}$ and Zhankui Jia ${ }^{1,2^{*}}$
}

\begin{abstract}
Background: ATP-binding cassette transporter super-family including ABCC1 and MDR-1 were involved in multidrug resistance (MDR) of renal cell carcinoma (RCC) patients. Several miRNAs were confirmed to promote the MDR and the survival of tumor cells.

Methods: The RCC cell lines Caki-2 with vinblastine-resistant (Caki-2/NBL) or doxorubicin-resistant (Caki-2/DOX) were constructed, respectively. The expressions of miR-210-3p, ABCC1 and MDR-1 protein were determined by qRT-PCR and Western blot assays. The viability of RCC cells was assessed by MTT assay. The regulatory relationship between miR-210-3p and ABCC1 was analyzed by Dual Luciferase assay. The effect of miR-210-3p in vivo was investigated with a tumor xenograft model in mice.

Results: MiR-210-3p expression was observed to significantly decrease in Caki-2NBL and Caki-2/DOX cells. Meanwhile, ABCC1 and MDR-1 were significantly increased in Caki-2/NBL and Caki-2/DOX cells. ABCC1 was a novel target of miR-210-3p and negatively regulated by miR-210-3p. And miR-210-3p improved drug-sensitivity of RCC cells.

Down-regulation of $A B C C 1$ could reverse the effect of miR-210-3p knockdown on the drug-resistance and the level of MDR-1 in drug-sensitive RCC cells.

Conclusion: We confirmed that down-regulation of miR-210-3p increased ABCC1 expression, thereby enhancing the MRP-1-mediated multidrug resistance of RCC cells.
\end{abstract}

Keywords: Renal cell carcinoma, Multi-drug resistance, MiR-210-3p, ABCC1, MDR-1

\section{Background}

Renal cell carcinoma (RCC) is one of the most lethal urologic malignancies worldwide with significant morbidity, mortality and poor prognosis [1]. The surgical therapy, including radical resection or nephron-sparing surgery, was commonly used as the preferred method for RCC. For those with advanced or recurring RCC patients, chemotherapy is the mainstream method for $\mathrm{RCC}$ in clinic, but it has unsatisfactory results in RCC patients [2]. The main reason for chemotherapy failure is that RCC cells develop multidrug resistance (MDR) to

\footnotetext{
*Correspondence: zhankuijia80@163.com

${ }^{1}$ Department of Urology, The First Affiliated Hospital, Zhengzhou University, No 1 Jianshe East Rd., Zhengzhou 450052, People's Republic of China

Full list of author information is available at the end of the article
}

chemotherapy agents, such as vinblastine and doxorubicin [3]. MDR expanded the ability of RCC cells to resist the cytotoxicity induced by chemotherapy agents, which was also accurately regulated by non-coding RNAs, proteins and signaling pathways [4]. The exploration of MDR mechanisms in RCC has become a new research direction in this field.

RCC patient with insensitivity to conventional chemotherapy agents may attribute to the intrinsic or acquired multi-drug resistance. ABCC1 and MDR-1, two numbers of ATP-binding cassette transporter super-family related to multi-drug resistance, were documented to increase in RCC patients and served as the efflux pumps to promote chemotherapeutic drugs out of cancer cells via the assistance of ATPase activity $[5,6]$. The expression of ABCC1 and MDR-1 could act as the MDR markers in RCC [6]. 
However, the relative contributions and causative roles of ABCC1 and MDR-1 in MDR of RCC cells have not been completely clarified.

MicroRNAs (miRNAs), a class of non-coding RNAs with the length of 18-25nt, are implicated in various fundamental biological processes and cancer pathological processes [7] through binding with $3^{\prime} U T R$ of target mRNAs, thereby causing the inhibition of translation and the degradation of mRNA, eventually to modulate gene expression at the post-transcriptional level [8]. More and more evidences have indicated that the aberrant miRNA expression is related to drug resistance/sensitivity and pathology of RCC $[9,10]$. MiR-210-3p was reported to be depleted by CRISPR/Cas9 to promote tumorigenesis through TWIST1 revival in RCC [11]. Moreover, miR210-3p was predicted to have the binding site on the 3'UTR of ABCC1. Hence, we hypothesized that miR210-3p was involved in the underlying mechanism of MDR in RCC.

\section{Methods}

\section{Cell culture and induction of drug-resistant cell lines}

Caki-2 cells, a human RCC cell line, were purchased from American Type Culture Collection (ATCC; Manassas, VA, USA) and were cultured in the McCoy's 5A medium (Thermo Fisher scientific, Massachusetts, USA) supplied with $10 \%$ FBS and $100 \mu \mathrm{g} / \mathrm{ml}$ double-antibody at $37{ }^{\circ} \mathrm{C}$ with the humidified $5 \% \mathrm{CO}_{2}$. Caki-2/DOX (doxorubicinresistant) and Caki-2/VBL (vinblastine-resistant) cells, the drug-resistant RCC cell lines, were constructed via Caki-2 cell lines (their independent parental cell lines) being exposed to the $\mathrm{IC}_{50}$ concentration of $\mathrm{DOX}$ and VBL for 3 months, and then exposed to tenfold higher dose of $\mathrm{IC}_{50}$ for 6 months with the same cultural conditions as Caki-2 cell lines [12].

\section{Cell transfection}

The RCC cell lines (Caki-2, Caki-2/DOX, and Caki-2/ VBL) were seeded in the 6-well plates with the density of $2 \times 10^{5}$ cells $/ \mathrm{ml}$, and then maintained for $24 \mathrm{~h}$. Caki-2 cells were transfected with miR-210-3p mimic/pre-NC $(20 \mathrm{nM})$ or miR-210-3p inhibitor $(50 \mathrm{nM})+$ si-ABCC1/ si-control $(20 \mathrm{nM})$ using Lipofectamine 2000 (Invitrogen, USA) following the manufacturer's protocol. Caki-2/ DOX and Caki-2/VBL cells were transfected with miR210-3p mimic/NC or miR-210-3p mimic + pcDNAABCC1/pcDNA by Lipofectamine 2000 (Invitrogen). The transfected RCC cells were maintained for $48 \mathrm{~h}$, followed by harvested for the next experiments. The detailed sequence information was as follows: miR-210-3p mimic, 5-CUGUGCGUGUGACAGCGGGUGA-3; miR-210-3p inhibitor, 5-UCAGCCGCUGUCACACGCACAG-3; si-ABCC1, 5-GUUCCAAGGUGGAUGCGAATT-3.
$\mathrm{ABCC} 1$ overexpression construct (pcDNA-ABCC1) was synthetized by Guangzhou RiboBio Co., Ltd (Guangzhou, China). The ABCC1 sequence was amplified with forward (F, 5-GTCGACACCATGGCCTGCTATTGC-3) and reverse ( $\mathrm{R}$, 5-GATGGATCCGCAGCAGAATGCCCAG-3) primers. After sequence validation, the sequences were subcloned into pcDNA3.1 vector.

\section{Quantitative real-time PCR}

The levels of miR-210-3p expression and ABCC1 mRNA expression were determined by quantitative real-time PCR (qRT-PCR). Total RNA was extracted from RCC cell lines using TRIzol (Invitrogen). The extracted RNAs were reverse transcribed to complementary DNA with the PrimeScript ${ }^{\circledR}$ RT reagent kit (TaKaRa). The levels of miR-210-3p expression and ABCC1 mRNA expression were quantified by SYBR $^{\circledR}$ Premix DimerEraser kit (TaKaRa) with 7500 Fast RealTime PCR System in the ABI Prism 7500 (Applied Biosystems). U6 was used as the internal control for miR-210-3p, and GAPDH acted as the internal control for $A B C C 1$. Comparative $C T$ method, $2^{-\Delta \Delta C t}$, serves as the calculation method of relative gene expression. The primer sequences used in qPCR were as follows: miR210-3p, forward 5-GTGCAGGGTCCGAGGT-3, reverse 5-TATCTGTGCGTGTGACAGCGGCT-3; MDR1, forward 5-CCCATCATTGCAATAGCAGG-3, reverse 5-TGTTCAAACTTCTGCTCCTGA-3; ABCC1, forward 5-ATGTCACGTGGAATACCAGC-3, reverse 5-GAAGACTGAACTCCCTTCCT-3; U6, forward 5-CT CGCTTCGGCAGCACA-3, reverse 5-AACGCTTCAC GAATTTGCGT-3.

\section{Western blotting}

The levels of ABCC1 and MDR-1 protein were assessed by Western blot assays in accordance with previous report [13]. Total protein from RCC cell lines was extracted with RIPA lysis buffer and then separated by SDS-PAGE on $10 \%$ acrylamide gels, followed by transferred into PVDF membrane. Afterwards, the membrane was incubated with primary antibodies against $\mathrm{ABCC} 1$ (1:1000 dilution, Abcam, Cat. no. ab99531), MDR-1 (1:500 dilution, Abcam, Cat. no. ab129450) and $\beta$-actin (1:3000 dilution, Abcam, Cat. no. ab8226) $\left(4{ }^{\circ} \mathrm{C}\right.$ overnight) and then maintained with HRP-conjugated secondary antibody for $1 \mathrm{~h}$. Protein bands were visualized with Amersham ECL Western blotting detection reagents (GE Healthcare, Piscataway, NJ, USA).

\section{Cell viability}

For drug-resistant analysis, the cell viability assays were performed. RCC cells $\left(2 \times 10^{4}\right.$ cells/well $)$ were planted in 96-well plates and cultured at $37{ }^{\circ} \mathrm{C}$ with a $5 \% \mathrm{CO}_{2}$ 
humidified atmosphere for $24 \mathrm{~h}$. Afterwards, DOX with various concentrations $(0,10,50,100,200,400 \mu \mathrm{g} / \mathrm{ml}$ and $0,1,2,3,4,5 \mu \mathrm{g} / \mathrm{ml}$ ) were respectively administrated to Caki-2/DOX and Caki- 2 cell culture, and VBL at various concentrations $(0,10,50,100,200,300$ and $0,1,2,3,4$, $5 \mu \mathrm{g} / \mathrm{ml}$ ) were respectively administrated to Caki-2/VBL and Caki-2 cell culture for $24 \mathrm{~h}$ of incubation. The MTT assays were applied to analyze the viabilities of each RCC cell line in accordance with previous report [14]. MTT solutions $(20 \mu \mathrm{l}, 5 \mathrm{mg} / \mathrm{ml})$ was added to each well for $4 \mathrm{~h}$ at $37^{\circ} \mathrm{C}$.

\section{Dual Luciferase assay}

The bind site of miR-210-3p and ABCC1 were predicted and the wild type (WT) and mutant (Mut; the bind site was mutant) fragment of ABCC1 was shown in Fig. 3a. Two fragments were amplified by PCR using the primers: for the WT segment, 5'-AATTAGATCTAAAGAAA AGCGAGAGCAGCA-3' (forward) and 5'-AATTAG ATCTGCTCTCTGGGTTTGAAGTCG- $3^{\prime}$ (reverse); for the Mut segment, 5'-AATTAGATCTGCTGTGA AGCACACGGAGAG-3' (forward) and 5'-AATT AGATCTCAGACATTCGCGGTCAGAGA-3' (reverse). Two $A B C C 1$ fragments were respectively cloned into the downstream of the luciferase gene of pGL3 Luciferase miRNA Target Expression Vector (Promega, Madison, WI, USA) to synthesize the recombinant reporter vector named pGL3-ABCC1-WT and pGL3-ABCC1-Mut. The reporter vector pGL3-ABCC1-WT/pGL3-ABCC1-Mut and miR-210-3p inhibitor/NC were co-transfected into Caki-2 cells using Lipofectamine 2000 (Invitrogen). The reporter vector pGL3-ABCC1-WT/pGL3-ABCC1-Mut and miR-210-3p mimic/pre-NC were co-transfected into Caki-2/DOX and Caki-2/VBL cells using Lipofectamine 2000 (Invitrogen). After positive lysis of the cells, multimode detector with the Dual-Luciferase Reporter Assay System (Promega) was used to evaluate the activities of luciferase.

\section{Xenograft model}

In order to analyze the effects of miR-210-3p on drugresistant renal tumor growth in vivo, a nude mouse tumor xenograft model was established. The present study was approved by Animal Care and Experimentation Committee of The First Affiliated Hospital of Zhengzhou University. Nude mice were transplanted subcutaneously with $2.5 \times 10^{6}$ Caki-2/DOX cells with/without miR210-3p over-expression into the right flank [pre-NC group $(\mathrm{n}=8)$ and miR-210-3p mimic group $(\mathrm{n}=8)]$. After 10 days, the mice of two groups were treated with DOX $(2 \mathrm{mg} / \mathrm{kg} /$ day $)$ via intraperitoneal injections. Every 3 days, the length $(\mathrm{L})$ and width (W) of tumor in mice were measured, and the tumor volume was calculated using the following equation: $\left(\mathrm{L} \times \mathrm{W}^{2}\right) / 2$. After 30 days, the mice were killed and tumor tissues were removed for the following study.

In the following experiment, Mice were transplanted subcutaneously with $2.5 \times 10^{6} \mathrm{Caki}-2$ cells with/without miR210-3p knockdown into the right flank [NC group $(\mathrm{n}=8)$ and miR-210-3p inhibitor group $(\mathrm{n}=8)]$. After 10 days, the mice of two groups were treated with DOX $(2 \mathrm{mg} / \mathrm{kg} /$ day $)$ via intraperitoneal injections. Every 3 days, the length $(\mathrm{L})$ and width $(\mathrm{W})$ of tumor in mice were measured, and the tumor volume was calculated using the following equation: $\left(\mathrm{L} \times \mathrm{W}^{2}\right) / 2$. After 30 days, the mice were killed and tumor tissues were removed for the following study.

\section{Statistical analysis}

All data from three independent repeated experiments were exhibited as the mean \pm SD and statistically analyzed with Student's $t$ test for single comparison between two groups and one-way ANOVA for comparison of multiple groups on SPSS 17.0 (SPSS Inc., Chicago, IL, USA). A value of P less than 0.05 was considered statistically significant.

\section{Results \\ The expression of miR-210-3p was decreased and the levels of ASCC1 and MDR-1 were increased in drug-resistant RCC cells}

The RCC cell line Caki-2 with vinblastine-resistant (Caki-2/VBL) or doxorubicin-resistant (Caki-2/DOX) were constructed, respectively. The expressions of miR210-3p (Fig. 1a), ABCC1 and MDR-1 protein (Fig. 1b, c) were determined. The results of qRT-PCR and Western blot assays showed that the expression of miR-210-3p was decreased and the levels of $A B C C 1$ and MDR-1 were increased in Caki-2/DOX and Caki-2/VBL cells, compared to the RCC cell line Caki-2 (drug-sensitive cells).

\section{The expression of miR-210-3p could decline the drug resistance of RCC cells}

MiR-210-3p levels were up-regulated in Caki-2/DOX and Caki-2/VBL cells via transfection with miR-210-3p mimic, followed by treated with different concentration of DOX and VBL, respectively. The viabilities of Caki-2/ DOX and Caki-2/VBL cells with miR-210-3p overexpression were declined, which suggested that up-regulation of miR-210-3p could elevate the drug-sensitivity of RCC cells (Fig. 2a). In addition, Caki-2 cells with miR210-3p knockdown were treated with different concentrations of DOX or VBL. The increased viability of Caki-2 cells indicated that the drug-resistance of RCC cells was enhanced by miR-210-3p down-regulation (Fig. 2b). 


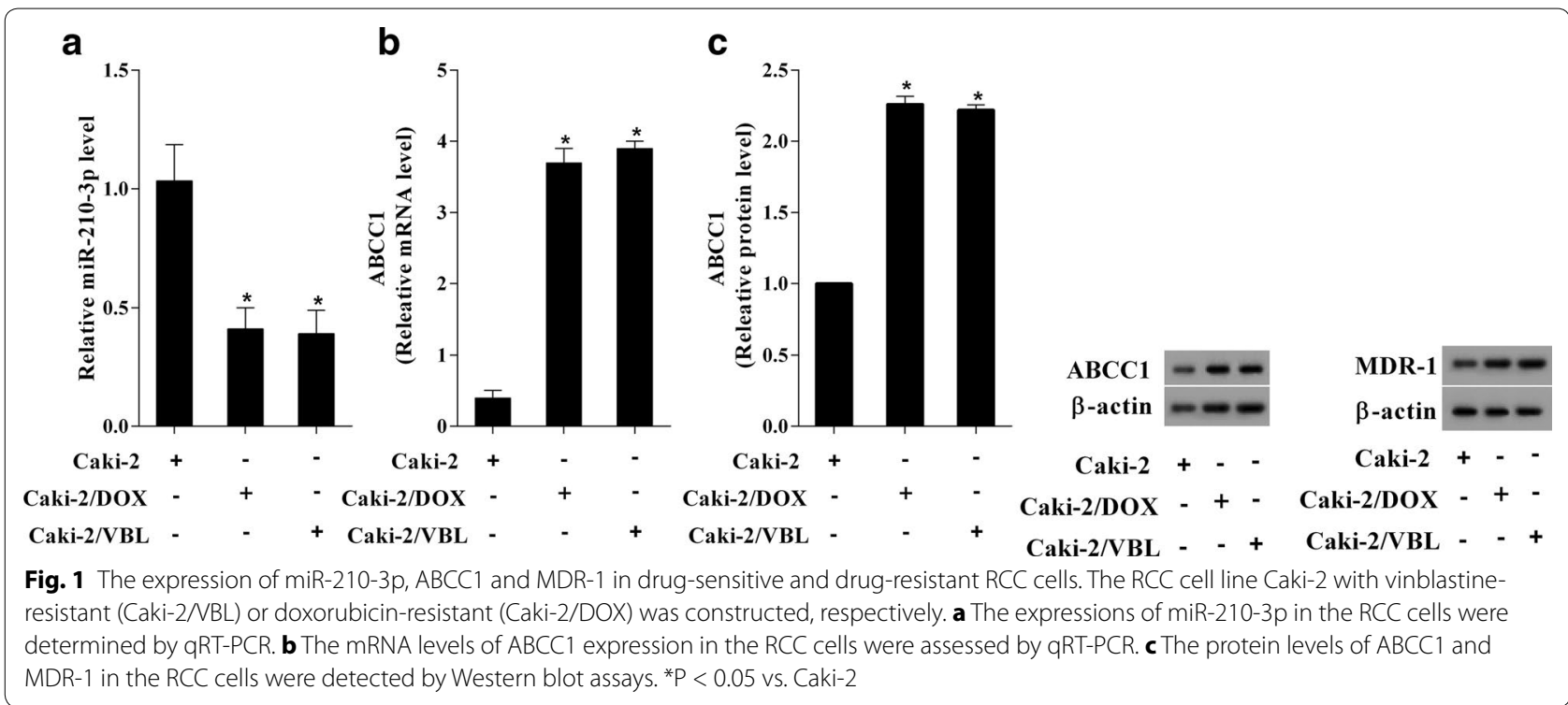

\section{MiR-210-3p could regulate the ABCC1 expression}

For evaluating the regulatory relationship between miR-210-3p and ABCC1, Dual Luciferase assays were performed on RCC cell lines Caki-2, Caki-2/DOX and Caki-2/VBL. As shown in Fig. 3a, bioinformatics software predicted that miR-210-3p had the bind site on the 3'UTR of ABCC1. The luciferase activity of Caki-2 cells was increased by co-transfection with pGL3-ABCC1WT and miR-210-3p inhibitor, and the luciferase activity of Caki-2 cells transfected with pGL3-ABCC1-Mut showed no difference after miR-210-3p knockdown. The expression of $A B C C 1$ was up-regulated by miR-210-3p down-regulation at both mRNA and protein levels (Fig. 3b). MiR-210-3p mimic and pGL3-ABCC1-WT/ pGL3-ABCC1-Mut were co-transfected into Caki-2/ DOX and Caki-2/VBL cells, and the luciferase activities in Caki-2/DOX and Caki-2/VBL cells was reduced by cotransfection with pGL3-ABCC1-WT and miR-210-3p mimic. Meanwhile, the expression of ABCC1 was inhibited by miR-210-3p over-expression at both mRNA and protein levels (Fig. 3c).

\section{MiR-210-3p modulated MDR-1 expression and drug-resistance of RCC cells via ABCC1}

After the drug-sensitive RCC cell line Caki-2 were transfected with miR-210-3p inhibitor, the level of MDR-1 in Caki-2 cell was enhanced. Then knockdown of ABCC1 in Caki-2 cell transfected with miR-210-3p inhibitor could reverse the effect of miR-210-3p down-regulation on the MDR-1 regulation (Fig. 4a). Meanwhile, the enhanced cell viability and drug-resistance induced by miR-210-3p knockdown were also reversed by $\mathrm{ABCC} 1$ inhibition in Caki-2 cell treated with different concentration of DOX or VBL (Fig. 5a). Moreover, the expression of miR-210-3p was up-regulated in the drug-resistant RCC cell lines, which obviously inhibited the level of MDR-1 in Caki-2/ DOX and Caki-2/VBL cells. Further, the decreased levels of MDR-1 expression induced by miR-210-3p overexpression were reversed by the up-regulation of $\mathrm{ABCC} 1$ in Caki-2/DOX and Caki-2/VBL cells (Fig. 4b). Similarly, ABCC1 over-expression could reverse the miR-210-3p over-expression-induced the decrease of cell viability and drug-resistance in Caki-2/DOX and Caki-2/VBL cells treated with different concentrations of $\mathrm{DOX}$ or $\mathrm{VBL}$ (Fig. 5b).

\section{MiR-210-3p promoted the drug-sensitivity of RCC in mice}

DOX was used to inject into the mice injected by Caki-2/DOX cells with/without miR-210-3p overexpression. The tumor volume was markly reduced in the mice of miR-210-3p mimic group, which exhibited that the miR-210-3p effectively enhanced the DOX-sensitivity of RCC to inhibit the growth of tumor (Fig. 6a). The levels of ABCC1 and MDR-1 were also declined in the mice of miR-210-3p mimic group (Fig. 6b). On the other hand, DOX was used to inject into the mice injected by Caki-2 cells with/without miR-210-3p inhibition. The DOX-resistance of RCC enhanced the speed of tumor growth in the mice of miR-210-3p inhibitor group (Fig. 7a). The levels of ABCC1 and MDR-1 were also remarkably elevated in the mice of miR-210-3p inhibitor group (Fig. 7b). 

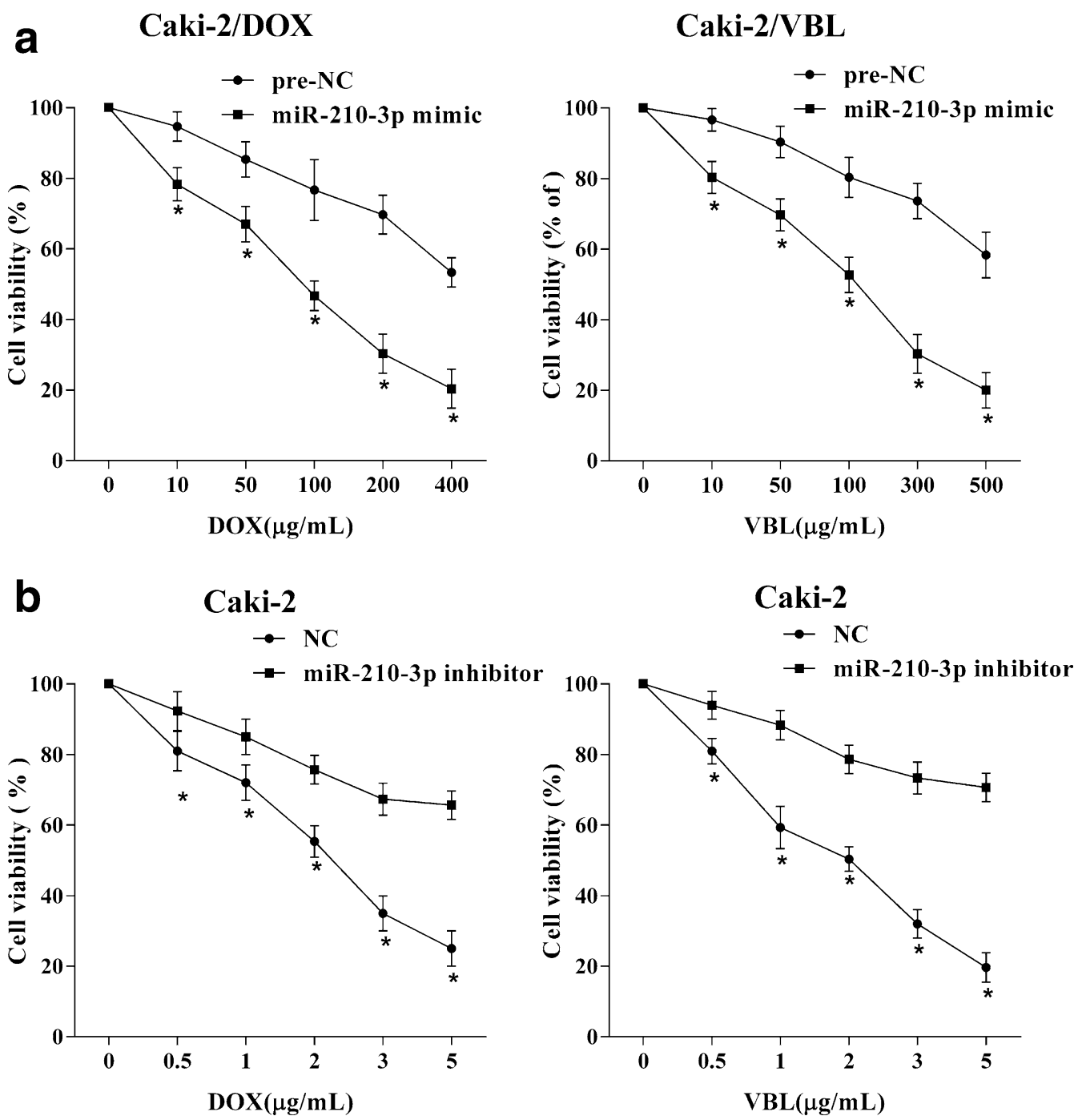

Fig. 2 The influence of miR-210-3p on drug resistance of RCC cells. a After Caki-2/DOX and Caki-2/NBL cells were transfected with miR-210-3p mimic and then respectively treated with $\operatorname{DOX}(0,10,50,100,200,400 \mu \mathrm{g} / \mathrm{ml})$ and VBL $(0,10,50,100,200,300 \mu \mathrm{g} / \mathrm{ml})$, followed by the cell viabilities were detected by MTT assays. ${ }^{*} \mathrm{P}<0.05$ vs. pre-NC. b After Caki-2 cells were transfected with miR-210-3p inhibitor and then respectively treated with DOX $(0,1,2,3,4,5 \mu \mathrm{g} / \mathrm{ml})$ or VBL $(0,1,2,3,4,5 \mu \mathrm{g} / \mathrm{ml})$, followed by the cell viabilities were detected by MTT assays. ${ }^{*} \mathrm{P}<0.05 \mathrm{vs}$. NC

\section{Discussion}

Chemotherapeutic unresponsiveness, metastatic spread and recurrence of RCC are mainly resulted from MDR. MiR-210-3p has been detected to be differently expressed between the drug-resistant and drug-sensitive RCC cells and involved in the drug-sensitivity of RCC cells. In our study, we focused on the role of miR-210-3p in the occurrence of RCC drug-resistance and further explored its underlying mechanism.

ATP-binding cassette transporter super-family has vital effect on MDR in cancer, which is reported to a main leading cause of chemotherapeutic failure in various cancers through regulating the efflux of chemotherapeutic drugs [15-18]. Previous reports have exhibited $\mathrm{ABCC} 1$ to be up-regulated in colorectal cancer [19], lung cancer [20], and breast cancer [21]. MDR-1 serving as a protein scavenger also can capture and transport various chemotherapeutic agents out of cells [22]. MDR-1 has been reported to be up-regulated in various chemotherapeutic-resistant cancer cell lines [23] and also be over-expressed in RCC patients [6]. The emergence of chemo-refractory with MDR greatly limits the efficacy 

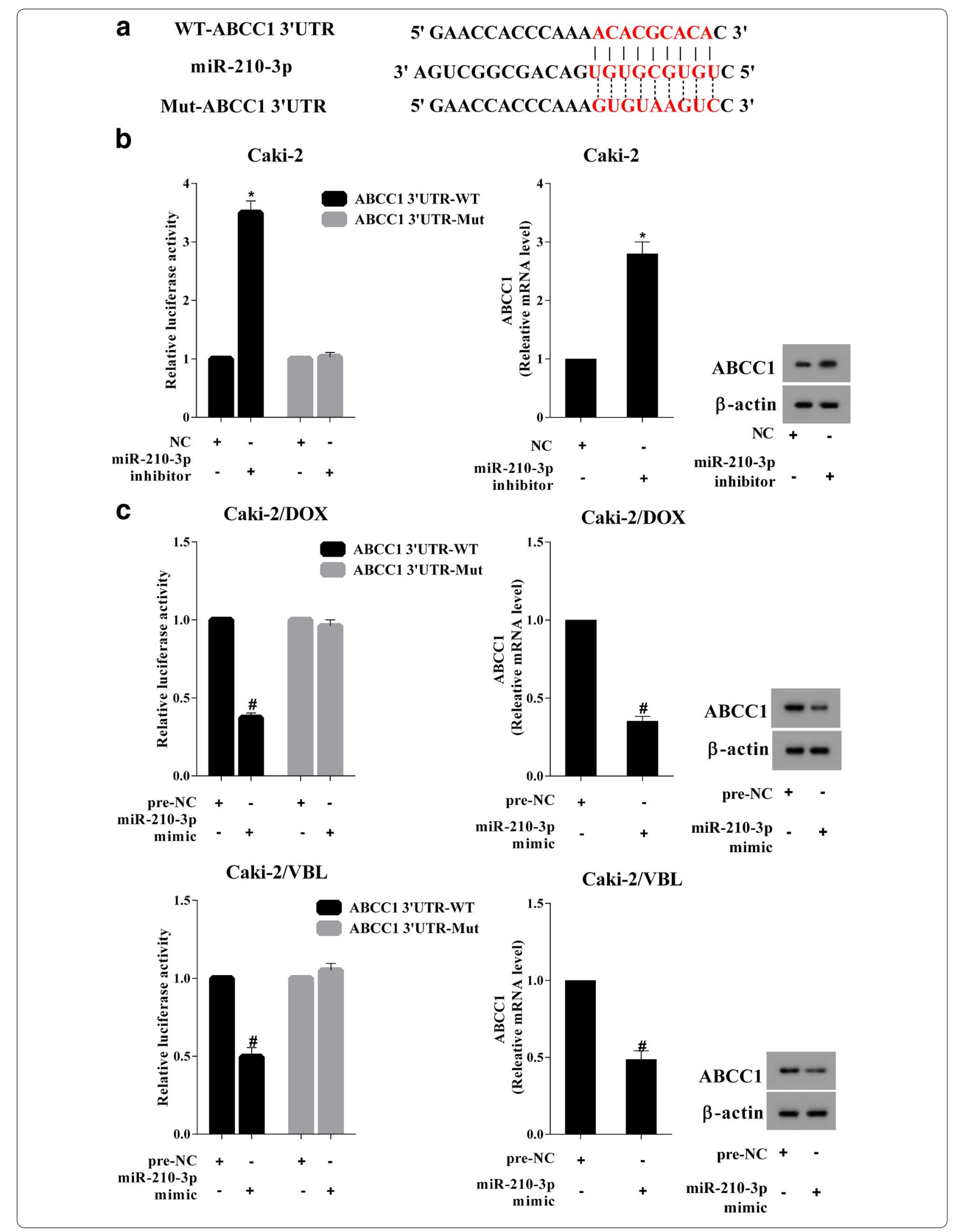
(See Figure on previous page.)

Fig. 3 The mechanism of miR-210-3p regulating ABCC1. a The binding site of miR-210-3p on ABCC1 3'UTR was predicted by bioinformatics software. $\mathbf{b}$ In the Caki-2 cells, knockdown of miR-210-3p up-regulated the $3^{\prime} U T R$ activity of wild type ABCC1 and the expression of ABCC1. c Overexpression of miR-210-3p inhibited the $3^{\prime} U T R$ activity of wild type $A B C C 1$ and the expression of ABCC1 in drug-resistant RCC cells. ${ }^{*} P<0.05$ vs. NC, $\# P<0.05$ vs. pre-NC

a

b

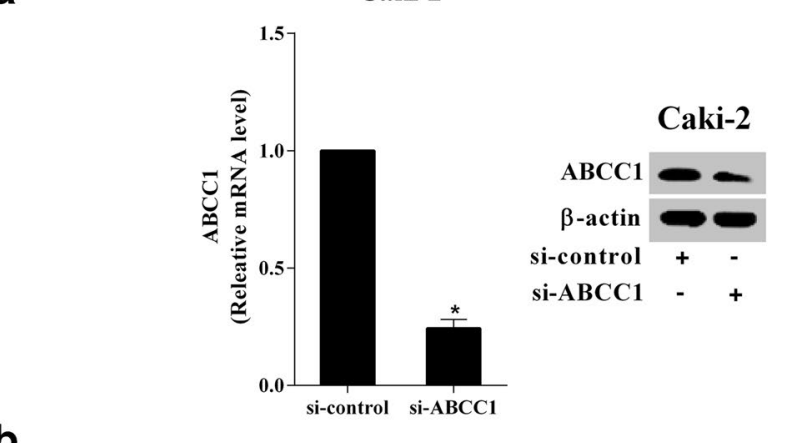

Caki-2/DOX
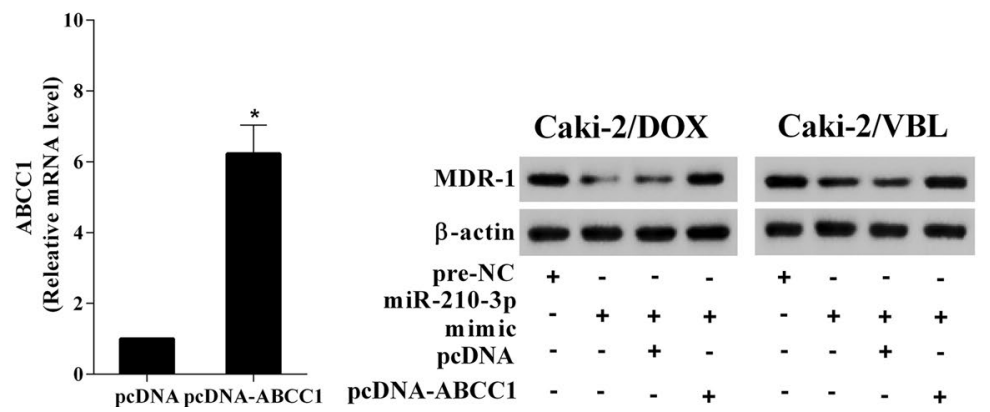

Caki-2

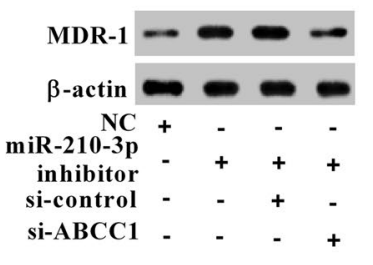

Fig. 4 MiR-210-3p modulated MDR-1 expressions of RCC cells via ABCC1. a Caki-2 cells were transfected with si-ABCC1 or si-control, the knockdown efficiency of ABCC1 was detected using qRT-PCR and Western blot assays. Caki-2 cells were transfected with miR-210-3p inhibitor or miR-210-3p inhibitor + si-ABCC1, and then the levels of MDR-1 were assessed by Western blot assays. b Caki-2/DOX and Caki-2/NBL cells were transfected with pcDNA-ABCC1 or pcDNA, the overexpression efficiency of ABCC1 was detected using qRT-PCR and Western blot assays. Caki-2/DOX and Caki-2NBL cells were transfected with miR-210-3p mimic or miR-210-3p mimic + pcDNA-ABCC1, and then the levels of MDR-1 were assessed by Western blot assays. ${ }^{*} \mathrm{P}<0.05$ vs. si-control or pcDNA

and application of broad-spectrum conventional tumor chemotherapeutics [24]. In our study, we also observed the up-regulation of $\mathrm{ABCC} 1$ and MDR-1 in DOX-resistant and VBL-resistant RCC cells.

Emerging researches have indicated that several miRNAs were significantly associated to the recurrence and survival of patients with RCC and might act as biomarkers for the diagnosis of RCC patients with high risk in early recurrence phase after surgical resection in kidney [25]. In like manner, miRNAs promoted the phenotype of drug-resistant and the survival of tumor cell via directly targeting MDR family members to regulate the multi-drug resistance. MiR-210-3p was reported to highly express in clinical ccRCC specimens (compared to adjacent non-cancerous tissues) and RCC cell lines 786-o, A498 and Caki-2 (compared to normal kidney cells). However, Yoshino et al. reported that the higher expression of miR210-3p found in the ccRCC clinical samples and the cell lines was probably inhibitory to tumor progression, as shown by an accelerated cell invasiveness and an increased number of colonies in the miR210-3p-depleted cells in comparison to the controls [11]. In the present study, the expression of miR-210-3p was decreased in Caki-2/DOX and Caki-2/VBL cells, compared to the RCC cell line Caki-2, suggesting the correlativity between miR-210-3p and drug-resistance of RCC cells. We further confirmed that miR-210-3p improved drug-sensitivity of RCC cells through inhibiting ABCC1. And up-regulation of miR-210-3p could decrease the drug-resistance and the levels of ABCC1 and MDR-1 in 

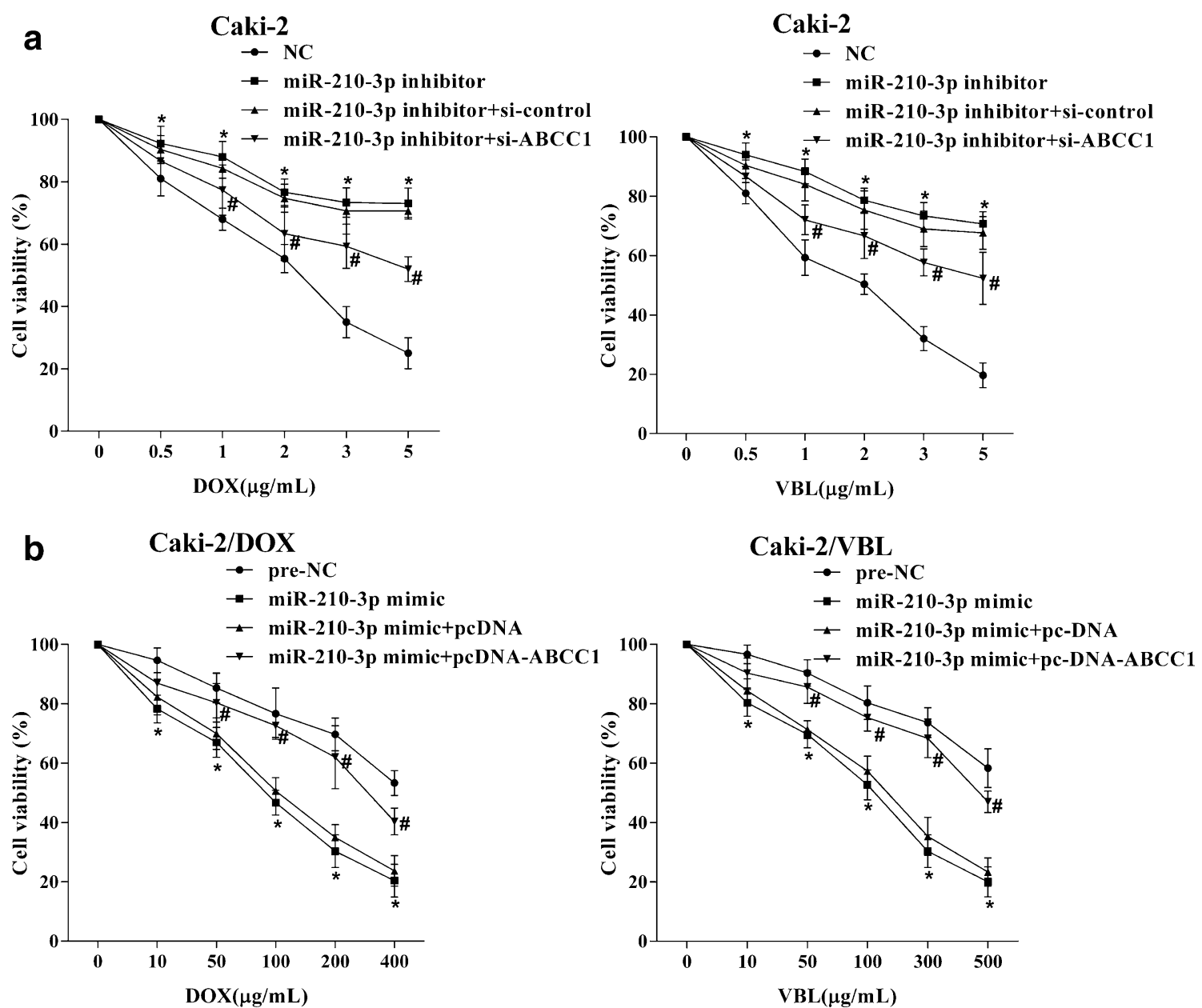

Fig. 5 MiR-210-3p modulated drug-resistances of RCC cells via ABCC1. a Caki-2 cells were transfected with miR-210-3p inhibitor or miR-210-3p inhibitor + si-control, and then cell viabilities were assessed by MTT assays. b Caki-2/DOX and Caki-2/NBL cells were transfected with miR-210-3p mimic or miR-210-3p mimic + pcDNA-ABCC1, then cell viabilities were assessed by MTT assays. ${ }^{*} \mathrm{P}<0.05 \mathrm{vs}$. NC or pre-NC, $\mathrm{P}<0.05 \mathrm{vs}$. miR-210-3p inhibitor + si-control or miR-210-3p mimic + pcDNA

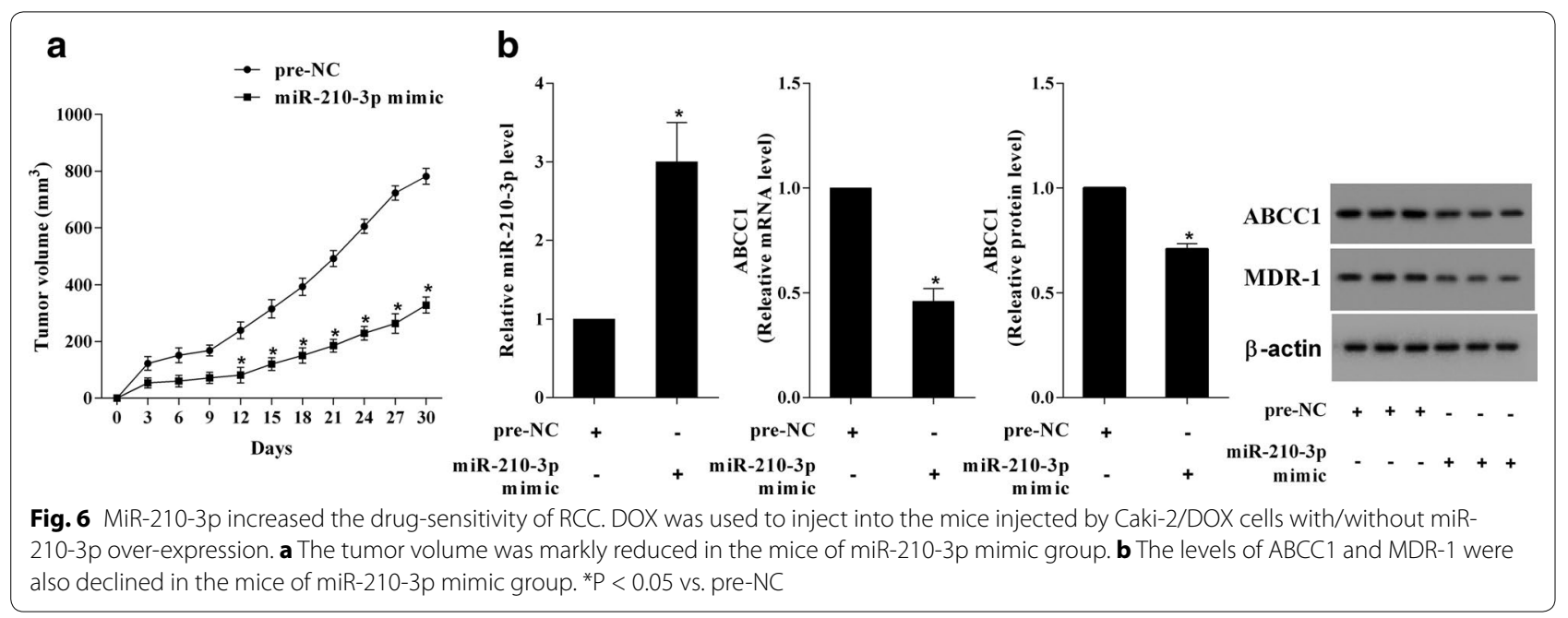




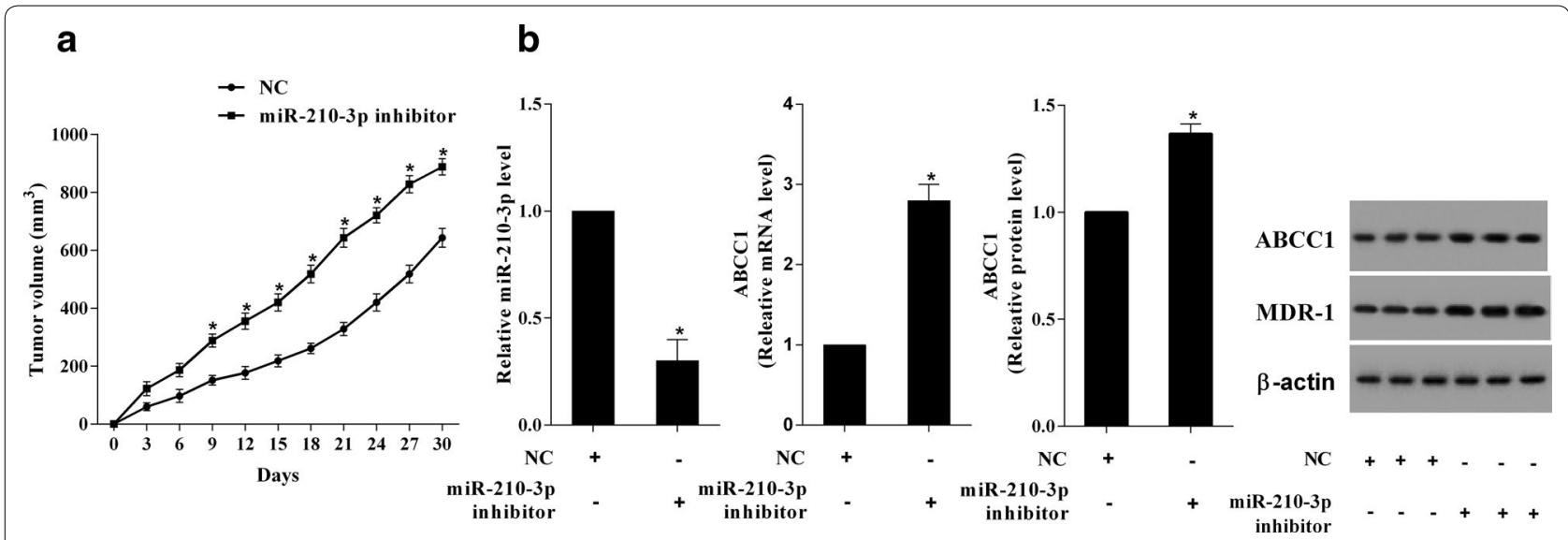

Fig. 7 MiR-210-3p decreased the drug resistance of RCC. DOX was used to inject into the mice injected by Caki-2 cells with miR-210-3p inhibition. a The tumor volume was markly enhanced in the mice of miR-210-3p inhibitor group. $\mathbf{b}$ The levels of ABCC1 and MDR-1 were also enhanced in the mice of miR-210-3p inhibitor group. ${ }^{*} \mathrm{P}<0.05$ vs. NC

drug-resistant RCC cells. Hence, we confirmed that miR210-3p mediated multi-drug resistance of RCC cells via binding with ABCC1. Moreover, miR-210-3p improved drug-sensitivity of RCC cells through inhibiting ABCC1. We identified the existence of miR-210-3p/ABCC1 axis in multidrug resistance of RCC cells, which also were proved in vivo.

In conclusion, we confirmed that down-regulation of miR-210-3p increased ABCC1 expression, thereby enhancing the MRP-1-mediated multidrug resistance of RCC cells, as shown by an increase in MDR1 expression and in cell viability with DOX or VBL treatment.

\section{Authors' contributions}

SCL put forward the concept of the study, designed the study, prepared the manuscript and contributed to the statistical analysis. JJY contributed to the data acquisition. JW and YFD contributed to the quality control of data and algorithms. WG and YHD analyzed the data and interpretation. ZKJ edited the manuscript. All authors read and approved the final manuscript.

\section{Author details}

1 Department of Urology, The First Affiliated Hospital, Zhengzhou University, No 1 Jianshe East Rd., Zhengzhou 450052, People's Republic of China. ${ }^{2}$ Urological Institute of Henan, Zhengzhou 450052, Henan Province, People's Republic of China.

\section{Acknowledgements}

Not applicable.

\section{Competing interests}

The authors declare that they have no competing interests.

\section{Availability of data and materials \\ Not applicable.}

\section{Consent to publish}

Not applicable.

\section{Ethics approval and consent to participate}

The study was approved by ethics committee of hospital.

\section{Funding}

This work is supported by the National Natural Science Foundation of China (Grant No 81400689).

\section{Publisher's Note}

Springer Nature remains neutral with regard to jurisdictional claims in published maps and institutional affiliations.

Received: 23 November 2017 Accepted: 31 January 2018

Published online: 07 February 2018

\section{References}

1. Jemal A, et al. Cancer statistics, 2004. CA Cancer J Clin. 2004;54(1):8-29.

2. Lim SH, et al. Intrinsic resistance to sunitinib in patients with metastatic renal cell carcinoma. Asia Pac J Clin Oncol. 2017;13(1):61-7.

3. van der Mijn JC, et al. Predictive biomarkers in renal cell cancer: insights in drug resistance mechanisms. Drug Resist Updat. 2014;17(4-6):77-88.

4. Long QZ, et al. miR-124 represses FZD5 to attenuate P-glycoproteinmediated chemo-resistance in renal cell carcinoma. Tumour Biol. 2015;36(9):7017-26.

5. O'Connor R. The pharmacology of cancer resistance. Anticancer Res. 2007;27(3A):1267-72.

6. Walsh $\mathrm{N}$, et al. Expression of multidrug resistance markers ABCB1 (MDR1/P-gp) and ABCC1 (MRP-1) in renal cell carcinoma. BMC Urol. 2009;9:6.

7. Ambros $V$. The functions of animal microRNAs. Nature. 2004;431(7006):350-5.

8. Feng DD, et al. Down-regulated miR-331-5p and miR-27a are associated with chemotherapy resistance and relapse in leukaemia. J Cell Mol Med. 2011;15(10):2164-75.

9. Gong J, et al. Microparticles and their emerging role in cancer multidrug resistance. Cancer Treat Rev. 2012;38(3):226-34.

10. Gowrishankar B, et al. MicroRNA expression signatures of stage, grade, and progression in clear cell RCC. Cancer Biol Ther. 2014;15(3):329-41.

11. Yoshino H, et al. microRNA-210-3p depletion by CRISPR/Cas9 promoted tumorigenesis through revival of TWIST1 in renal cell carcinoma. Oncotarget. 2017:8(13):20881-94.

12. Long QZ, et al. Interaction of CCN1 with alphavbeta3 integrin induces $P$-glycoprotein and confers vinblastine resistance in renal cell carcinoma cells. Anticancer Drugs. 2013;24(8):810-7.

13. Liu J, et al. PRDM5 expression and essential role after acute spinal cord injury in adult rat. Neurochem Res. 2016;41(12):3333-43. 
14. Wu DD, et al. MicroRNA-873 mediates multidrug resistance in ovarian cancer cells by targeting ABCB1. Tumour Biol. 2016;37(8):10499-506.

15. Qiu JG, et al. Trametinib modulates cancer multidrug resistance by targeting ABCB1 transporter. Oncotarget. 2015;6(17):15494-509.

16. Vilanova-Costa CA, et al. MDR1 and cytochrome P450 gene-expression profiles as markers of chemosensitivity in human chronic myelogenous leukemia cells treated with cisplatin and Ru(III) metallocomplexes. Biol Trace Elem Res. 2015;163(1-2):39-47.

17. Hung $T H$, et al. Wnt5A regulates $A B C B 1$ expression in multidrug-resistant cancer cells through activation of the non-canonical PKA/beta-catenin pathway. Oncotarget. 2014;5(23):12273-90.

18. Zhou $X$, et al. Dichloroacetate restores drug sensitivity in paclitaxel-resistant cells by inducing citric acid accumulation. Mol Cancer. 2015;14:63.

19. Chen M, et al. miR-133b down-regulates ABCC1 and enhances the sensitivity of CRC to anti-tumor drugs. Oncotarget. 2017:8(32):52983-94.
20. Pei K, et al. MicroRNA-185-5p modulates chemosensitivity of human non-small cell lung cancer to cisplatin via targeting ABCC1. Eur Rev Med Pharmacol Sci. 2016;20(22):4697-704.

21. Spitzwieser $M$, et al. Promoter methylation patterns of $A B C B 1, A B C C 1$ and $A B C G 2$ in human cancer cell lines, multidrug-resistant cell models and tumor, tumor-adjacent and tumor-distant tissues from breast cancer patients. Oncotarget. 2016;7(45):73347-69.

22. Gisel A, et al. miRNAs for the detection of multidrug resistance: overview and perspectives. Molecules. 2014;19(5):5611-23.

23. Zhou SF. Structure, function and regulation of $P$-glycoprotein and its clinical relevance in drug disposition. Xenobiotica. 2008;38(7-8):802-32.

24. Perez-Tomas R. Multidrug resistance: retrospect and prospects in anticancer drug treatment. Curr Med Chem. 2006;13(16):1859-76.

25. Slaby $\mathrm{O}$, et al. Identification of MicroRNAs associated with early relapse after nephrectomy in renal cell carcinoma patients. Genes Chromosomes Cancer. 2012:51(7):707-16.

\section{Submit your next manuscript to BioMed Central and we will help you at every step:}

- We accept pre-submission inquiries

- Our selector tool helps you to find the most relevant journal

- We provide round the clock customer support

- Convenient online submission

- Thorough peer review

- Inclusion in PubMed and all major indexing services

- Maximum visibility for your research

Submit your manuscript at www.biomedcentral com/submit 